\title{
7
}

\section{Chlamydia trachomatis Infections in Neonates}

\author{
Eszter Balla and Fruzsina Petrovay \\ National Center for Epidemiology \\ Hungary
}

\section{Introduction}

Chlamydia trachomatis is the most commonly reported agent among sexually transmitted bacteria in Europe and in the US (CDC, 2011; ECDC, 2011). In 2009 the constantly growing number of notified cases in Europe exceeded 340 000. This is presumably far below the true figure due to the asymptomatic nature of infections, which often remains undiagnosed. Genital C. trachomatis infections (serotype D-K) are especially common among sexually active young people (between 15 and 25 years of age), who represent as many as $75 \%$ of all reported infections across Europe (ECDC, 2011). Adolescents are threatened by acute urogenital manifestations (urethritis, epididymitis, cervicitis, etc.), that may turn into chronic complications (PID, infertility, ectopic pregnancy, etc.). The wide range of infections of the female genital tract poses a major reproductive health problem both on an individual and a community level.

Neonates of infected mothers form another important risk-group as women may transfer chlamydia not only to their sexual partners but if pregnant, to their infants as well. This vertical type of transmission is a well known phenomenon, which may result in ocular or in respiratory tract infection. Neonatal $C$. trachomatis infections include descending types of manifestation of syndromes, beginning with inclusion conjunctivitis, and/or nasopharyngal colonisation, which may be followed by neonatal pneumonia. These infections are generally mild-moderate in the case of an early and adequate treatment. The definitive diagnosis is hampered by the common phenomenon of asymptomatic, therefore often undiagnosed maternal infection, as well as by the need for adequate sample-collection and targeted screening techniques.

Neonatal infections due to vertical transmission have a so called "indicator" function too, namely, that they point to the infected mother and her sexual partner/s. It means that this kind of infections has a great epidemiological importance so the diagnostic and therapeutic efforts should be extended to cover all the exposed persons. Several specialists may be involved in this process, among them neonatologists, gynaecologists, urologists too. Unfortunately this seems to be a neglected area of neonatal infectology and there is only estimated data regarding the incidence of neonatal C. trachomatis infection, although all the necessary diagnostic means are available to identify the affected neonates. Focused screening efforts should be made to reduce the number of infected pregnant women and thereby the rate of vertical transmission. 


\section{Microbiology - Pathogenesis}

Chlamydia trachomatis is an obligate intracellular pathogen. This species is currently divided into 19 serovars based on the antigenic difference between the major outer membrane proteins (MOMP) and numerous serovariants differentiated with genotyping by restriction fragment length polymorphism (RFLP) analysis of the PCR-amplified omp1 gene. Urogenital, thereby neonatal infections are predominantly related to the D-K serotypes, while the A-C and L serotypes are not significantly associated with the latter group. Main target sites of the pathogen are the uro-anogenital organs of adults, the respiratory tract of neonates and the conjunctivae in both age groups. These local, non-invasive infections are typically limited to the epithelial surface of the mucous membranes.

The main transmission route involves sexual contact where due to the infected genital secretion a direct transfer of the pathogen occurs, which leads to the common clinical manifestations of uro-anogenital infection, however auto- or heteroinoculation may seldom result in conjunctivitis. Untreated maternal C. trachomatis carriage represents a special case of multiple risks threatening pregnant women themselves, their sexual partners as well as their infants.

Infants commonly acquire the pathogen by vertical transmission, typically during transit through the infected birth channel at delivery, however there are many reports suggesting intrauterine $C$. trachomatis infection. Besides the indirect evidence of a heightened risk of adverse pregnancy outcomes associated with $C$. trachomatis, the presence of $C$. trachomatis DNA in the fetal membranes detected by PCR confirms the intrauterine infection theory. A suspected choriodecidual inflammation and histological chorioamnionitis can give a possible explanation for the mechanism that may lead to the premature rupture of amniotic membranes. Chorioamnionitis is frequently seen in the case of premature babies, but the role of chlamydial infection in subsequent respiratory insufficiency is yet to be determined (Blas et al, 2007; Givner et al, 1981; Mårdh, 2002; Numazaki, 2004; Rastogi et al, 1999, 2003; Yoshida et al, 2000).

\section{Epidemiology - Transmission rates}

Due to the clinically unapparent nature of C. trachomatis infection in adults the reported cases allow only an estimation of its true prevalence. High risk groups may serve as a hidden reservoir of the pathogen, where the infection can easily spread through untreated carriers. A complex STI surveillance and screening programmes are required to gain more accurate information. Epidemiological studies may help to assess the role of this agent in various clinical pictures. Genital chlamydia infection rates among sexually active women in Europe are predominantly in the range of $4-10 \%$, which varies by age, socio-economic background and geographical location (Pellowe \& Pratt, 2006; Wilson et al., 2002).

In the United States the overall $C$. trachomatis prevalence among sexually active females aged 14-19 years was found to be $6,8 \%$, which is nearly three times the rate among those aged 25-39 years. There are significant differences between the various socioeconomic, ethnic and racial groups as well (CDC, 2011). It is a proven fact that young age $(<25$ years) and an improvident sexual behavior (new or multiple partners; unsafe sex, infection with another sexually transmitted disease, etc.) are associated with an increased risk of infection, which may also be attributable to other physiological/hormonal factors, such as an 
immature cervix, cervical friability/ectopy, or alteration in the cervical mucus (Peipert, 2003).

In a recent, population-based study C. trachomatis prevalence was found 3,9\% among more than 4,000 pregnant women, while during a previous, five-year period study $4,7 \%$ of 5531 pregnant women proved to be infected (Rours et al, 2011; Schachter et al, 1986). The rate of occurrence among infants is directly related to the maternal carriage of the pathogen, and strongly depends on the perinatal prevention strategies. The estimated data of neonatal prevalence rates range from 4 to 60 per 1000 live births depending on the population tested (Zar, 2005), which makes this microorganism the most frequent sexually transmitted pathogen in the industrialised countries, although detailed surveillance reports are nowadays still lacking. In a prospective study focusing on asymptomatic neonatal chlamydial colonisation and on symptomatic infection, the incidence per 1000 live birth was found to be 6,5 and 8,2 respectively, a summarised value of those represents a $14,7 \%$ prevalence of all chlamydia-positive infants (Preece et al, 1989).

The direct contact transmitting the pathogen derives sometimes from the infected nursing persons, mainly from family members or hospital staff or other care-takers, whose contaminated fingers or towels, etc. may spread chlamydiae to the infants. However rare this route of the infection may be, it should always be considered when investigating the source of neonatal infection (Mårdh, 2002).

Vertical transmission rates are relatively high as $50-75 \%$ of infants born vaginally acquire $C$. trachomatis directly from their infected mothers (Chen et al, 2007; Rours et al, 2008). One or more organs of neonates may be colonised, including the nasopharynx as the leading target site. Conjunctivae and the lower anogenital tract are less frequently involved, however the rectal and vaginal persistance may last as long as 3 years (Schachter et al, 1979). Chronic infection of the conjunctivae due to an untreated neonatal condition was also described, while the isolation of the pathogen was successful even at age of six years (Thompson et al, 2001). Most of these latter cases remain symptomless, but that fact may have medico-legal importance and must be carefully explored. From a clinical point of view only $20-50 \%$ of colonisations will result in acute conjunctivitis, and approximately 5-20\% in neonatal pneumonia, respectively (Darville, 2005; Numazaki et al, 1989; Schachter et al, 1986).

Recent studies suggest that prenatal transmission rates due to an ascending intrauterine infection seem to be significantly lower than those with vaginal delivery. Chorionic villus sampling completed with PCR technique can reveal C. trachomatis infection even in early pregnancy, when the incidence of chorioamnionitis related to the pathogen proved to be 5,1\% (Dong et al, 1998). Another sampling possibility targets the amniotic fluid, which may be investigated by serology, antigen- or DNA detecting methods too. There is data about the presence of a specific C. trachomatis antigen in 3,8\% of the amniotic samples (Djukić et al, 1996). There are documented cases of neonatal chlamydial infections after caesarean deliveries with ruptured membranes, and more rarely with intact membranes (Mohile et al, 2002). The prevalence of $C$. trachomatis infection among newborns after a caesarean section was found to be 8,3\% (Bell et al, 1994; Preece et al, 1989; Yu et al, 2009). These data indicate that intrauterine $C$. trachomatis infections can occur in any phase of the pregnancy, however at a relatively low risk. 


\section{Antenatal complications of maternal C. trachomatis infection}

Infected pregnant women and their fetuses are threatened by various complications resulting from ascension of pathogens into the upper genital tract and invasion of the amniotic cavity. Bacterial exposition can lead to infection at any time during pregnancy. Adverse pregnancy outcomes related to $C$. trachomatis include spontaneous abortion, premature rupture of membranes (PROM), preterm delivery and postpartum endometritis (Baud et al, 2008; Brocklehurst \& Rooney, 2007; Ostaszewska-Puchalska et al, 2005).

The actual cause of spontaneous abortion often remains unexplained. Besides chromosomal, immunologic or hormonal abnormalities, organic or functional disorders, an infective etiology may also be presumed. Controversial data is available regarding the role of $C$. trachomatis but recent studies seem to confirm it. In a prospective study investigating the presence of specific DNA in aborted tissues prevalence of $C$. trachomatis was found to be a surprisingly high 32\% (Magoń et al, 2005).

Prenatal infection is thought to be a relevant cause of preterm deliveries in up to $40-48 \%$ of all cases, that may occur either spontaneously or in form of a premature rupture of membranes (PROM) (Baud et al, 2008; Lamont et al, 1987; Locksmith \& Duff, 2001; Maxwell, 1993). Ascending microbial invasion of placenta, fetal membranes, and amniotic fluid is associated with an intense maternal inflammatory response that may eventually trigger a fetal inflammatory response. This pathological process is histologically characterised by funisitis and/or chorioamnionitis, caused by the same microbes as those isolated from the lower genital tract of pregnant women (Rezeberga et al., 2008). Chorioamnionitis is found in overall $30-70 \%$ of preterm births, while only in $4-18 \%$ of full term deliveries (Locksmith \& Duff, 2001). Despite often being a subclinical condition, chorioamnionitis may facilitate the weakening and rupture of membranes. The main problem with histologic diagnosis comes from the fact that the sampling of the placenta and fetal membranes can be carried out only after delivery. The unfavourable consequences of placental inflammation are not yet completely understood, but direct impact of pathogens or organ damage due to inflammatory cytokines and proteolytic enzymes may both serve as an explanation. Several surveys indicate that among other bacteria deriving from urogenital reservoir $C$. trachomatis carriage also increases the risk of the premature rupture of membranes (PROM), as well as that of preterm uterine activity in infected women, which may contribute to fetal morbidity and mortality (Blas et al, 2007; Mårdh, 2002, Yu et al; 2009). Much of the harmful complications of $C$. trachomatis infection are supposed to occur via immunopathological damage (Baud et al, 2008).

C. trachomatis infection was found to be responsible for several perinatal complications, such as low-birth-weight and prematurity, defined as being born before 37 completed gestational weeks (Gencay et al, 2001; Rastogi et al, 1999). Chlamydial etiology was proven in preterm infants suffering from severe acute respiratory distress within the first day of life, requiring mechanical ventillation and supplemental oxygen (Colarizi et al, 1996). It was documented being associated with chronic respiratory disease in premature infants as well, while the presence of specific IgM suggested intrauterine infections during late pregnancy (Mårdh, 2002; Numazaki et al, 1986, 2003). Since it occasionally has a serious impact on the fetus, prenatal $C$. trachomatis infection should be prevented by comprehensive screening programs during pregnancy. 


\section{Postnatal clinical manifestations in C. trachomatis infected neonates}

There are two main target sites of the pathogen in affected neonates, which are responsible for the principal clinical manifestations of infection, accordingly ocular and respiratory tract infections are among the commonest neonatal complications. The possible association between inclusion conjunctivitis and subsequent infantile pneumonitis was first documented in a case report in 1975 (Schachter et al, 1975). Due to direct exposure to the infected maternal genital secretion, conjunctivae may be easily colonized by C. trachomatis, followed by an early onset of ocular symptoms, while respiratory tract infections due to aspiration of the bacteria generally show a descending pattern, deriving from an acute/subacute nasopharyngitis and resulting later in a clinical condition of pneumonia. The relatively frequent conjunctivitis, nasopharyngitis and the less common pneumonia, as the most serious complication often may combine, and these associated symptoms refer to the stepwise progression of the infection. A retrospective study analysing the occurrence rate of various clinical appearances found that conjunctivitis was present in $73 \%$, pneumonia in $20 \%$ and both diseases in $7 \%$ of affected newborns. Hospitalisation was needed in $20 \%$ of infants (Jain, 1999). Other, less frequent clinical findings include rhinitis, otitis media, while myocarditis and encephalitis have been documented as rare, unusual manifestations of the infection.

\subsection{Conjunctivitis, or ophthalmia neonatorum}

Neonatal conjunctivitis is one of the most common types of infections in the first 30 days of life. It is defined as an inflammation of the conjunctivae that may be caused by various toxic or infectious agents. Chlamydiae as obligate intracellular agents can intensely replicate in conjunctival cells, which leads to epithelial damage. In case of acquiring $C$. trachomatis (serotypes D-K) the ocular infection manifests as a so called inclusion conjunctivitis, when during intracellular development cycles of chlamydiae infectious elementary bodies of the pathogen differentiate into large, metabolically active reticulate bodies. The latter accumulate in the endosomes of host cells turning them into cytoplasmic inclusions, which can be detected after Giemsa staining even by a conventional light microscope. During the inflammatory response, granulocytes invade the site of infection, which appears clinically in the form of a mucopurulent exudate. The untreated infection resolves spontaneously in most patients during a couple of months, however, a formation of a micropannus (granulation tissue-membrane) or of a pannus (corneal neovascularizaton) may sometimes occur. Infrequently persistent conjunctivitis and trachoma-like corneal/conjunctival scarring have been documented too (Darville, 2005; Quirke \& Cullinane, 2008).

C. trachomatis has been regularly reported as a leading agent for neonatal conjunctivitis, or ophthtalmia neonatorum in several studies analysing bacterial etiology. There are some surveys which still support that prominent position by investigating certain populations (Rours et al, 2008; Salpietro et al, 1999; Schaller \& Klauss 2001). It is an interesting fact that the pathogen itself was first isolated precisely from a neonatal conjunctivitis in 1959 (Jones et al, 1959). In the 1980's C. trachomatis was detected in as many as $14-46 \%$ of neonatal conjunctivitis, however, the latest studies estimate the descending prevalence of $C$. trachomatis as being approximately $8 \%$ in this condition. Its significant role moreover seems to be recently extinguished by some classical pyogenic bacteria, such as Staphylococcus 
aureus, Haemophilus influenzae and Streptococcus pneumoniae (Di Bartolomeo et al, 2001; Persson et al, 1983; Valencia et al, 2000).

The results of studies implemented in various countries clearly reflect that the distribution of major etiological agents of neonatal conjunctivitis is in accordance with the actual prevalence of STI pathogens among pregnant women. In examination of newborns in the United Arabic Emirates less than 5\% of all cases were attributable to $C$. trachomatis and Neisseria gonorrhoeae. C. trachomatis alone was responsible in Norway for only $6 \%$, while in India and in Kenya for $24 \%$ and $28,7 \%$ of all neonatal conjunctivitis respectively (Dannevig et al, 1992; Mohile et al, 2002; Nsanze et al, 1996; Schaller \& Klauss, 2001). In the Netherlands chlamydial screening of pregnant women is not a routine practice, which may play a significant role in high rates of $C$. trachomatis detection, which was calculated over $60 \%$ of studied infants presenting conjunctivitis (Rours et al, 2008). In the 1980's the incidence of chlamydial ophthalmia neonatorum was found 3 - 250 cases per 1,000 live births, which has radically declined to 0,65 per 1,000 live births in Europe during the last three decades. This decreasing trend can be particularly observed in developed countries, where screening and treatment during pregnancy is a regular part of C. trachomatis surveillance (Darville, 2005; Di Bartolomeo et al, 2001; Krohn et al, 1993; Persson et al, 1983; Preece et al, 1989; Quirke \& Cullinane, 2008).

In contrast with classical trachoma caused by A-C serotypes, those responsible for neonatal eye infection belong to the D-K serotypes and correlate with the actual distribution of urogenital maternal carriage of the pathogen (Di Bartolomeo et al, 2001; Isobe et al, 1996). Some data suggest that certain genotypes may be strongly asssociated with ophthtalmia neonatorum, as an epidemiological link was recently found between genotype $\mathrm{E}$ and the ocular symptoms (Gallo Vaulet et al, 2010). The perinatal transmission rate of infection resulting in conjunctivitis is relatively high, as the risk of a newborn acquiring $C$. trachomatis in case of maternal carriage varies between $8-44 \%$. Based on pooled results of previous studies, the point estimate of incidence of ophthalmia neonatorum proved to be $15 \%$ among infants exposed to C. trachomatis perinatally (Rosenman et al, 2003). The incubation period usually lasts between 5-14 days after delivery, which may be shorter in the case of an intrauterine infection due to PROM. The median age of onset of the ocular symptoms is typically 1-2 weeks after birth. That means that the signs of infection acquired intrapartum will usually develop after leaving hospital, generally up to one month of age (Miller, 2006; Persson et al, 1983). Affected newborns would have most often been seen and treated by two or more physicians before establishing the definitive diagnosis of $C$. trachomatis infection. In case it is recognised promptly and treated adequately, a complete recovery can be expected without any late complications (Preece et a, 1989). Misdiagnosis or mistreatment with topical and/or inadequate medications will just facilitate the persistence of the disease. Due to the slowly progressive nature of the untreated condition, it can be only occasionally diagnosed in infants after two months of age (Mårdh, 2002; Rours et al, 2008).

Neonatal chlamydial conjunctivitis generally starts mono-ocularly, but after 2-7 days the progressive involvement of the other eye can be observed in $75 \%$ of the newborns. Clinical signs can widely vary and range from mild conjunctival injection to serious exsudative ophthalmitis. Classifying the severity of the symptoms, more than half of the detected cases proved to be moderate, while a serious condition was found in almost $37 \%$ of the patients in an extended survey (Stenberg \& Mårdh, 1990). Conjunctival erythema, palpebral edema, 
chemosis and scanty discharge are typical in this condition. The triad of hyperemia, swelling and discharge should always be evaluated as a warning sign, however, there are some infected newborns who do not show this complete clinical picture. Initially the exudate may be watery, turning later mucoid, or pucopurulent. In the case of an intense inflammation, the massive exudate adheres to the conjunctivae and so evolves a characteristic pseudomembrane. Everted conjunctivae may bleed at examination or at sampling procedure. Bloody discharge may occasionally develop, which has a particular diagnostic value being highly specific for $C$. trachomatis. Rhinitis, as a frequently associated sign was observed in $60 \%$ of newborns suffering from chlamydial ophthalmia (Dannevig et al, 1992). Most untreated cases tend to spontaneous recovery and do not threaten the vision of the affected babies, but may occasionally persist for a longer period accompanied by superficial corneal vascularisation and conjunctival scarring. Despite the prescribed treatment the persistance of ocular infection was observed in $12 \%$ of patients, confirmed by the reisolation of $C$. trachomatis, which may refer to an inadeqaute therapy or lack of compliance. The most serious late complication manifests as visual loss due to trachoma-like tarsal scarring and pannus formation. Blindness develops in this chronic condition much slower than compared to gonococcal conjunctivitis (Darville, 2005; Mårdh, 2002; Persson et al, 1983; Rees et al, 1981; Quirke \& Cullinane, 2008; Zar, 2005).

The differential diagnosis of chlamydial ophthtalmia neonatorum is assisted by the knowledge of potential etiological agents, however, in the majority of patients the actual cause remains obscure. "Red eye” as an early symptom often develops at newborns, and besides infection it may be evoked by trauma or by chemical irritation due to some topical medications. Redness and purulent discharge related to silver-nitrate instillation usually starts on the first day of life, but lasts only for a few days. However, this clinical condition may be associated with several microorganisms, and the significance of those has varied over time. Infectious origin pertains predominantly to bacteria apart from the easily recognizable but rare herpes simplex virus infection. Based on etiology studies neither other viruses, nor genital mycoplasmas play any significant role in the pathogenesis (Krohn et al, 1993; Prentice et al, 1977). When the conjunctivitis persists beyond three days and is not healed by the use of a frequent normal saline irrigation, then one should immediately start empiric treatment before getting laboratory results indicating some kind of infection.

Main bacterial pathogens including C. trachomatis, Neisseria gonorrhoeae and other pyogenic species can be identified only in $35-60 \%$ of the cases (Di Bartolomeo et al, 2001; Jarvis et al, 1987; Krohn et al, 1993; Pierce et al, 1982). In a well-designed study in the Netherlands an uncommonly high detection rate was documented with $84 \%$ of identified pathogens (Rours et al, 2008). Chlamydial ophthalmia may be occasionally superinfected with other agents, which may make the accurate diagnosis more difficult. In complicated, therapy-resistant cases one should consider the presence of $N$. gonorrhoeae or of other nosocomial pathogens as well (Winceslaus et al, 1987). The elimination of the disease may be interfered not only by secondary infections, but by the recurrence of the original agent as well. In a follow-up study the recurrence rate of chlamydial neonatal conjunctivitis was found as much as $35 \%$, which raises questions about therapeutic efficacy and compliance (Mohile et al, 2002).

$N$. gonorrhoeae was once the commonest cause of ophthtalmia neonatorum leading to irreversible corneal damage and subsequent blindness in several cases, which had caused 
major health problems in many countries for centuries before Credé's prophylaxis was introduced in 1881. Since Credé's time the prevalence of sight-threatening gonorrhoeal conjunctivitis among newborns has radically decreased due to comprehensive prevention efforts. As a result, $N$. gonorrhoeae now counts as a rare ocular isolate. Some surveys could detect not a single case of gonococcal ophthalmia among newborns (Dannevig et al, 1992; Prentice et al, 1977; Solberg et al, 1991). Clinically the symptoms of a N. gonorrhoeae infection would begin much earlier than those of $C$. trachomatis infection, as the former usually starts 2-5 days after birth with a severe purulent discharge that tends to rapid progression leading occasionally to corneal penetration and endophthalmitis (Quirke \& Cullinane, 2008). Just as in case of other pyogenic bacteria, the definitive laboratory diagnosis is based on traditional culture of the exudate, however the examination of a Gram-stained smear may be very informative and allows a presumptive, rapid detection of pathogens (Winceslaus et al, 1987).

Common expected pathogens further include Staphylococcus aureus, Streptococcus pneumoniae, Haemophilus influenzae, Pseudomonas aeruginosa, members of the Enterobacteriaceae family and other Gram-negative bacteria. The altered distribution pattern of pathogens indicates that $S$. aureus took over the leading role of previous agents in purulent conjunctivitis since it can be isolated in approximately 50\% of this condition (Dannevig et al, 1992; Nsanze et al, 1996; Sandström, 1978; Solberg et al, 1991). Being a nosocomial infection, staphylococcal conjunctivitis usually derives from the hospital environment and it is characterised by a more massive purulent discharge and a milder injection than chlamydial conjunctivitis (Darville, 2005; Prentice et al, 1977; Schaller \& Klauss, 2001). Nonchlamydial, nongonorrhoeal bacterial pathogens seem to account for a considerable proportion of neonatal conjunctivitis that can not be fully prevented by usual ocular prophylaxis. As the spectrum of potential pathogens is not completely determined yet more research is needed in this field.

Partly pre-, partly postnatal managements can be used to prevent ophthalmia neonatorum. The targeted screening of pregnant women will be discussed later, as this clinical condition cannot be handled as a separate syndrome. Postnatal prophylaxis regarding neonatal conjunctivitis however still remains an important issue. The introduction of Credé's silvernitrate solution dramatically changed the prevalence, and subsequently the distribution of major ocular pathogens, as it was highly active against $N$. gonorrhoeae strains. Due to the systematic and extended application of $1 \%$ silver-nitrate ophthalmic drops, gonococcal ophthalmia neonatorum has become uncommon by now in the industrialised part of the world where its incidence decreased to 0,04 per 1,000 live births. However its toxic and irritating side-effect, which may induce a chemical conjunctivitis, did not make it an optimal medicament.

In comparison, the topical application of a $0,5 \%$ erythromycin- or 1,0\% tetracycline ointment was just as effective as silver-nitrate in preventing gonococcal conjunctivitis, except for the emerging resistant strains, but none of them proved to reduce the incidence of ophthalmia neonatorum due to $C$. trachomatis. The failure rate of antichlamydial prophylaxis in the case of erythromycin was calculated to be $7-19,5 \%$ (Black-Payne et al, 1989). Other antibiotics, such as chloramphenicol, neomycin or gentamicin have been widely applied too, but there is no convincing data about their efficacy (Assadian et al, 2002). A widespread use of topical antibiotics furthermore may always carry the risk of increasing antimicrobial resistance. 
Accordingly, the local administration of any of these drugs does not offer an adequate protection against chlamydial conjunctivitis, and cannot either eliminate the accompanying nasopharyngeal chlamydial colonisation (Dannevig et al, 1992; Miller, 2006; Quirke \& Cullinane, 2008; Salpietro et al, 1999; Zar, 2005). The failure of the complete eradication of chlamydiae may result in long-term ocular complications, such as recurrent or persistent conjunctivitis (Rees et al, 1981).

The application of a less toxic, antiseptic $2,5 \%$ povidone-iodine solution, which has a special antichlamydial effect as well, may offer a reasonable and cheap alternative in the developing countries with a high prevalence rate of N. gonorrhoeae (Isenberg et al, 2003; Schaller \& Klauss, 2001; Zar, 2005). Another relatively new, promising agent may be fusidic acid, but there is insufficient data supporting its prophylactic value (Zuppa, 2011). In the industrialised areas though, depending on the actual prevalence values, Credés classic prophylactic method seemes to be unnecessary any more, so its value should be reconsidered (Darling \& McDonald, 2010; Silva et al, 2008). As it happens, it was the same idea that has resulted in the discontinuation of general prophylaxis against ophthalmia neonatorum in some countries (Schaller \& Klauss, 2001). The main problem with postnatal prophylaxis regarding $C$. trachomatis conjunctivitis lies in that there are no topical medications which would be able to eradicate chlamydiae from all colonised sites, including the inner respiratory organs of a newborn's organism (Ratelle et al, 1997). Focusing on newborns who are threatened by proven maternal chlamydial carriage the benefits of oral erythromycin prophylaxis were weighed against the risk of pyloric stenosis, which is a potential, recently confirmed adverse effect of the treatment. Until the recognition of this association The Committee on Infectious Diseases of the American Academy of Pediatrics had recommended a 14-day course of oral erythromycin for all neonates exposed to $C$. trachomatis at delivery. Based on analysis of the results of this selective prevention programme only a close observation of these infants was suggested. Oral erythromycin treatment is recommended now exclusively for those newborns who develop symptoms of infection (Rosenman et al, 2003). In the absence of a harmless, even systematically effective ideal drug, prevention depends on the prenatal screening and/or on the early treatment of a manifestating $C$. trachomatis infection.

\subsection{Respiratory tract infections}

Based on the actual age of the patient neonatal pneumonia can be classified as early or late onset. Some authors suggest the first week of life for separating the two main groups associated with different etiology, while intrauterine acquired pneumonia can be ranged as a special subgroup among early onset conditions (Duke, 2005). This type of classification can be applied to respiratory tract infections caused by $C$. trachomatis too, because this pathogen can affect either the fetus or the newborn characterised by a distinct clinical presentation due to different pathogenesis. Ascending chlamydial infections in pregnant women may result in intrauterine complications, such as chorioamnionitis and PROM. Subsequent transplacental infections occur predominantly in cases with ruptured membranes, and less frequently with intact membranes (Mohile et al, 2002; Preece et al, 1989). Affected fetuses are at a higher risk of being born preterm and of developing respiratory distress symptoms early after birth, though this is a rare kind of vertical transmission and an uncommon 
congenital condition due to C. trachomatis (Boo, 2008; Colarizi et al, 1996; López-Hurtado et al, 1999; Sollecito et al, 1992; Zar, 2005).

The pathogenesis of most neonatal pulmonary cases rather involves the aspiration either of the infectious genital secret of the mother or that of the ocular discharge, draining from the lacrimal duct as a kind of extension of a concomitant conjunctivitis. These may be the direct or indirect colonisation routes leading to the pharyngeal presence of $C$. trachomatis, which is the most frequent target site detected in about $70-77 \%$ of the infected neonates (Darville, 2005; Stenberg \& Mårdh, 1990) and that serves as an infectious focus giving rise to recurrent conjunctivitis or pneumonia. Pharyngitis occasionally may persist up to 2 years of age or beyond (Bell et al, 1992; Mårdh, 2002; Rees et al, 1981, Zar et al, 1999). Fortunately, this type of colonisation remains often subclinical, as its progression to pneumonia is observed only in $30 \%$ of the cases (Chen et al, 2007). Based on pooled published data point estimate of incidence of pneumonia was calculated $7 \%$ among infants exposed to $C$. trachomatis perinatally. The estimated rate of hospitalisation is $20 \%$ among newborns suffering from pneumonia, which makes it the most significant complication of C. trachomatis infection (Rosenman et al, 2003).

The early onset type of the chlamydial respiratory distress syndrome in very preterm infants was characterised by a biphasic clinical manifestation showing a transient improvement, but followed by a significant worsening of the respiratory status, when also apneic spells were noted. All the affected patients needed assisted ventilation with oxygen therapy. In this second phase chest X-rays showed hypoexpansion and the reticular pattern of the lungs, while conjunctival and respiratory tract's samples proved to be positive for $C$. trachomatis (Sollecito et al, 1992). Severe congenital pneumonia due to C. trachomatis may even lead to fatal consequences in very preterm babies (25-32 weeks of gestation) (Attenburrow \& Barker, 1986). Respiratory failure may affect primarily very preterm, low birth-weight infants, but there are documented cases of these also among near term babies or in untreated neonatal pneumonia (Herieka \& Dhar, 2001, Zar et al, 1999). C. trachomatis infection has been associated with seriously chronic, occasionally lethal forms of pulmonary disorders of ventilated preterm neonates. Despite the occasional presence and specific antibody response its etiologic role in bronchopulmonary dysplasia (BPD) has however not yet been confirmed (Attenburrow \& Barker, 1986; López-Hurtado et al, 1999; Numazaki et al, 1986; Zar, 2005).

The "classical" chlamydial pneumonia belongs to late onset, mild to moderate neonatal diseases, as in full-term babies the symptoms of a $C$. trachomatis infection acquired intranatally usually onset between 2 and 19 weeks, typically around 6 weeks of age (Duke, 2005; Marín Gabriel et al, 2004; Pellowe \& Pratt, 2006). That relative delay may be explained by the time that is needed for the newborns to develop cellular immunity (Mårdh, 2002). Concomitant conjunctivitis can be detected in as many as half of the cases and may act as an important diagnostic clue (Darville, 2005). Tympanic membrane involvement seemes to be an infrequent accompanying symptom in about only $6 \%$ of the patients, however, even recurrent episodes of otitis media have been described among infants under 6 months exposed to C. trachomatis (Numazaki, 1984; Schaefer et al, 1985). Generally minor and aspecific clinical signs are present, such as subfebrility and slight tachypnoea, which cause a difficulty in recognising the condition. More than $95 \%$ of the infected newborns are afebrile. Rhinitis may be present in $20-67 \%$ of infected neonates, predominantly associated with 
conjunctivitis. Rhinorrhoea as a prodromal symptom with a duration of one-two weeks often precedes the lower respiratory tract signs, such as tachypnoea and persistent cough (Chen et al, 2007; Iskandar \& Naguib, 1998; Numazaki et al, 1989). Staccato-like coughing and congestion may however point to the possibility of a respiratory tract infection, while expiratory wheezes are uncommon (Klein \& Barnett, 1998). Persistent cough may interfere with feeding, moreover the nutrition problems have been significantly linked to the respiratory complication (Pellowe \& Pratt, 2006; Rours 2008; Zar, 2005).

The diagnosis is supported by crepitant inspiratory rales on auscultation and chest X-rays showing hyperinflation and diffuse bilateral, usually interstitial infiltrates. Generalised nodular shadowing has also been documented (Herieka \& Dhar, 2001). The radiographic image may show a more serious condition than suggested by the physical examination (Darville, 2005; Miller, 2006). Retraction and cyanosis have also been described among the conspicuous clinical signs of respiratory distress as well, observed primarily in the youngest newborns under one month of age (Numazaki, 1984; Preece et al, 1989). In the majority of the patients peripheral eosinophilia contributes to abnormal, but nonspecific laboratory results, suggesting that it has a significant diagnostic value (Chen et al, 2007; Darville, 2005; Mårdh, 2002; Numazaki, 1984).

Recognising and differentiating a chlamydial neonatal pneumonia is hampered by multiple difficulties, such as aspecific symptoms, the high number of potential other pathogens, the possibility of a superinfection and the various transmission routes. The infectious source of the neonatal pneumonia can be present in the maternal birth canal, in the hospital/nursery environment or at home. That means that the broad etiologic spectrum of infant pneumonitis extends from perinatally colonising agents via nosocomial pathogens to community acquired microbes (Klein \& Barnett, 1998). Several viruses, such as human respiratory syncytial virus (RSV), adenovirus, cytomegalovirus and bacteria, such as Streptococcus agalactiae, Staphylococcus aureus, Streptococcus pneumoniae, Bordetella pertussis, Ureaplasma species etc., and a parasite (Pneumocystis carinii) have been documented to be responsible for the condition. However, in most studies the actual cause of pediatric pneumonia could not be established in $40-60 \%$ of the cases, while mixed infections may contribute to further diagnostic problems (Jadavji, 1997). Multiple pathogens were generally identified in 24-27\% of the patients. Concurrent infections are typically characterised by more severe clinical signs, measured by the occurrence of apnea and the need for oxygen therapy and mechanical ventilation (Chen et al, 2007; Ejzenberg et al, 1996; Mårdh, 2002; Numazaki et al, 1989; Stagno et al, 1981).

In the developing countries $C$. trachomatis is accepted as one of the leading causes of late neonatal pneumonia, where approximately half of the cases were attributable to this pathogen (Were et al, 2002). The clinical significance of $C$. trachomatis is supported by the fact that recently it has still been detected as the second most frequent respiratory pathogen of infants aged less than 6 months after the human respiratory syncytial virus in the Netherlands (Rours et al, 2009). Also in another study RSV proved to be the most prevalent agent in as many as $44 \%$ of the patients suffering from lower respiratory tract infections (Vieira et al, 2003). The clinical manifestation of C. trachomatis infection may be indistinguishable from RSV infection, however, the latter usually follows a seasonal pattern, and is more often associated with fever and characteristic wheezing due to airway obstruction (Darville, 2005; Duke, 2005; Preece et al, 1989). Cytomegalovirus tends to affect 
other organs as well, while adenovirus or parainfluenzavirus infections are not linked with eosinophilia and staccato cough. Pyogenic bacteria, like Streptococcus agalactiae, Staphylococcus aureus, Streptococcus pneumoniae or Enterobacteriaceae species etc. typically cause more severe, febrile, occasionally septic symptoms with distinctive radiologic signs. Bordetella pertussis is a rare respiratory pathogen of newborns known to develop characteristic signs of an intense paroxysmal cough with an inspiratory whoop, often followed by emesis. In its most serious form it may affect the unvaccinated, vulnerable age groups, especially the infants born prematurely (de Greeff et al, 2010). Ureaplasma urealyticum far more frequently colonises the airways of preterm premature infants than $C$. trachomatis, as Ureaplasma strains could be detected by PCR in $45 \%$, while less than $2 \%$ of those proved to be infected by $C$. trachomatis. The long-term sequelae however may not differ from each other because Ureaplasma carriage has been reported to correlate significantly with the development of bronchopulmonary dysplasia (BPD) (Da Silva et al, 1997; Garland \& Bowman, 1996).

\subsection{Rare organ manifestations and complications of C. trachomatis infection}

Neonatal encephalitis due to C. trachomatis was described in an immunodeficient infant, where the presence of the pathogen was confirmed in the tracheal aspirate and in the cerebrospinal fluid as well. Neurological symptoms followed the onset of neonatal pneumonia and may be associated with the defect of the alternative complement pathway (Bertsche et al, 2008). Infectious myocarditis was reported in an infant suffering from chlamydial pneumonitis. There have been only a few reported cases of childhood myocarditis in the literature associated with $C$. trachomatis infection, so heart muscle cells act as an especially rare target site (Odeh \& Oliven, 1992; Ringel et al, 1983).

Uro-anogenital colonisation occurs usually at birth though the carriage of the pathogen may asymptomatically persist for a long time. However the presence of $C$. trachomatis in the rectal or the genital area in a child over 3 years of age should always be evaluated as a potential warning sign of sexual abuse (Darville, 2005; Schachter et al, 1986). Without evidence of child sexual abuse other transmission routes should be considered, as it is reported in a case of a family cluster of $C$. trachomatis infection (Thompson et al, 2001).

Extragenital persistance of untreated neonatal C. trachomatis infections may last even longer, occasionally up to 6 years. The nasopharynx, the oropharynx and the conjunctivae are described to possible sites of such an enormous duration of colonisation. The cumulative proportion of untreated or mistreated infants still colonised at the age of 1 year was reported as many as 35\% (Bell 1992; Mårdh, 2002), which may serve as explanation when sexual assaults of the affected child can be definitely excluded.

A recent study confirmed the presence of both C. trachomatis and C. pneumoniae organisms in the lungs of patients suffering from chronic pulmonary disease, including asthma and other inflammatory airway disease. Corticosteroids have also been confirmed to reactivate persisting chlamydiae, so the combination of both inhaled and oral steroid treatment might act as a triggering factor of increased reactivation of chlamydiae contributing to asthmatic or other symptoms. Due to that phenomenon the latent respiratory carriage of these pathogens may turn into symptomatic disorder. The exact role of the Chlamydiaceae 
family is not fully understood yet so further research is needed concerning the etiology and exacerbation of paediatric asthma and other chronic respiratory diseases (Webley et al, 2009).

\section{Laboratory diagnosis}

According to its clinical and epidemiological relevance targeted diagnostic tests should include C. trachomatis for all infants aged less than 6 months who have suspicious clinical signs of ophthalmia neonatorum and/or pneumonia or other organ manifestations. The potential etiological role of $C$. trachomatis should be especially considered in countries with a high prevalence of the pathogen among young women and/or in the absence of an adequate screening programme in pregnancy. The topical use of antibiotics should be judged as an unreliable, insufficient mode of prevention. The correct laboratory results depend on the collection of relevant samples done by clinicians and on the use of the proper diagnostic test chosen by the microbiologists.

\subsection{Sample collection}

Conjunctivitis: Specimens for direct detection tests should be obtained from the everted eyelid using a Dacron-tipped swab or an equivalent recommended by the test kit manufacturer. As chlamydiae are obligate intracellular bacteria, it is important to collect some conjunctival cells from the inner surface of the eyelid, instead of sampling only the ophthalmic exudate. The latter procedure otherwise may lead to false-negative laboratory results. Serology is not informative because in the absence of a systemic immune response there are no detectable IgM levels in this localised type of infection (Mahony et al, 1986; Miller, 2006).

Pneumonia and other respiratory tract infections: In case of rhinitis or pharyngitis, specimen rich in mucosal cells should be collected from the affected upper air ways. In suspected otitis media the middle ear effusion is the optimal sample for confirming the presence of pathogen. From patients suffering from pneumonia respiratory tract samples should be collected, preferably from the nasopharyngeal area, acting as a common infectious source. However, the more relevant lower respiratory tract samples such as tracheal aspirates, lavage fluid, pleural fluid, bronchoscopic or lung biopsy specimens should also be definitely tested if they are available. Native blood or serum samples should be always enclosed, as one may expect a specific immune response in these conditions. According to some previous studies, the testing of cord blood samples may also be informative (Gencay et al, 2001).

Additional source tracking: Proven neonatal infections always indicate a human reservoir in the close environment, as C. trachomatis is a relatively sensitive micoorganism transferred by infectious secretions predominantly from urogenital or some other mucosal focus. Supposing vertical transmission, the maternal urogenital screening for $C$. trachomatis is suggested. Possible positive results represent a diagnosed infection, being often asymptomatic in adults, moreover it may confirm the infectious source of the neonatal condition. In the event that the maternal tests are negative, but there are verified positive neonatal laboratory results, other routes of transmission should be considered, and the screening efforts should be extended to other persons from the family or the nursing 
environment. Serology is unnecessary, because only the direct detection methods can be informative about acute urogenital infections.

\subsection{Laboratory methods}

\subsubsection{Direct detection techniques}

Direct detection techniques historically were limited to tissue culture, as conventional culture methods do not enable the isolation of this obligate intracellular pathogen. For optimal results tissue culture requires the presence of viable microorganisms, so the clinical specimen should contain not only exsudate but epithelial cells as well from the infected area. Besides appropriate sampling technique adequte transport media are also needed for maintaining the cultivability of the bacteria. Culture testing for $C$. trachomatis has always been the reference standard against which all the laboratory tests developed later have been compared. However, it can not be used for routine diagnostic purposes because of being difficult to standardize, technically demanding, time-consuming and expensive. This relatively insensitive method (its sensitivity ranges between $70-85 \%$ ) is available only in specialized laboratories but maintains its advantage in forensic cases of suspected sexul abuse, where it may provide conclusive evidence of a sexually transmitted infection (Johnson et al, 2002; Schachter \& Stamm, 1995).

Newer, non-viability dependent tests place less demands on specimen transport. The antigen detection tests applied most frequently rely either on the identification of chlamydial elementary bodies using fluorochrome-labelled, specific monoclonal antibodies (direct fluorescent antibody, or DFA test) or on the capture and detection of chlamydial antigen using enzyme immunoassay based techniques (enzyme immunoassay, or EIA). These two basic methods are widely used, being quite simple to perform and evaluate. However they are not considered "gold standard" diagnostic procedures any more, because in the meantime nucleic acid-based methods (hybridization-probes and amplification techniques) have been developed, which generally have more advantages.

Although at a greater cost and with a greater requirement for trained staff and technical conditions, the nucleic acid-based methods generally offer superior sensitivity and specificity, while non-invasively taken urogenital samples (urine for example) may count as adequate specimen as well. Nucleic acid amplification techniques (NAAT) include polymerase chain reaction (PCR), ligase chain reaction (LCR), strand displacement amplification (SDA) and transcription mediated amplification (TMA). The key advantage of multiplex molecular method is that they have the ability to detect other coinfecting pathogens (eg. Neisseria gonorrhoeae) in a single specimen as well. They also give rise to further typization tests such as restriction fragment length polymorphism (RFLP) analysis of the PCR-amplified omp1 gene used for differentiating the genotypes, that may have epidemiological importance contributing to surveillance studies (Petrovay et al, 2009).

\subsubsection{Serology}

The demonstration of specific antibodies in adults is rarely indicative for an acute urogenital infection of $C$. trachomatis, its use may be rather limited to only chronic, ascending infections. 
Other superficial mucosal infections, such as chlamydial conjunctivitis of patients of any age will not develop a systemic immune response either. However, when investigating neonates with pneumonia, the special circumstances of the condition (high probability of the actual presence of the pathogen in the upper respiratory tract, and the simultaneously detectable immune response) allow the completion of the laboratory tests with serological assays. Due to the high number of circulating B cells, plasma cells and large amounts of secreted antibodies, serology has become a crucial tool of laboratory tests in neonatal pneumonia. Sera of Japanese infants proved to be posivite for IgM antibodies to C. trachomatis in $29 \%$ of patients with pneumonia, furthermore in $66 \%$ of this subgroup even the direct detection technique could confirm the presence of the pathogen from nasopharyngeal samples. All the positive laboratory results corresponded with the clinical signs of neonatal pneumonia (Numazaki, 1984).

The results of several studies suggest that neonatal pneumonia may be accurately diagnosed by the detection of high IgM antibody titers in serum. The maternal IgM do not pass across the placenta, so the presence of $C$. trachomatis IgM antibody on newborns' blood samples unambiguously refers to neonatal infection, which proves to be a quite reliable sign of a systemic $C$. trachomatis infection, moreover, it may already be present at birth. In such cases as these IgM antibody titers in serum generally rise quickly after birth and approach maximal titers approximately by the second week of life and may persist for up to 3 months (Mahony et al, 1986). The intrauterin transmission of the pathogen has revealed that not only common serum samples taken postnatally but even cord blood samples of infected infants may contain specific IgM (Gencay et al, 2001; Numazaki et al, 1986).

Due to the antigenic relationship between various chlamydia species the cross reaction may frequently result in unreliable results while using tests of low-medium grade specificity. The highest specificity can be achieved by choosing a micro-immunofluorescence test (MIF) and the diagnostic criteria are adapted to elevated levels of $C$. trachomatis IgM detected by MIF. The cut-off value was defined as a titer $\geq 1: 16$ which is strongly suggestive of $C$. trachomatis infection (Numazaki, 1984). The use of other serological methods, such as ELISA tests are not recommended for that purpose. Due to the high occurance rates, the routine testing for $C$. trachomatis is needed in hospitalised infants having pneumonia.

\subsection{Results and evaluation}

Between January 2004 and June 2011 we examined the serum samples of 282 infants suffering from respiratory tract infection by C. trachomatis MIF (FOCUS) test in our laboratory (Second Department of Bacteriology, National Centre for Epidemiology, Budapest). The majority of the samples $(92,6 \%)$ were taken from infants younger than 20 weeks, which corresponds to the fact that this condition develops mainly in the first five months. The detailed results indicate that newborns aged 0-4 months represent more than $\mathbf{8 4} \%$ of all patients with a proven $C$. trachomatis infection. Positive IgM results were found altogether in 95 patients $(33,7 \%)$ in $\geq 1: 16$ titers. The positivity rate of our survey provided similar data to other reported findings, where the $C$. trachomatis infection was detected in approximately $30 \%$ of patients (Chen et al, 2007; Numazaki, 1984). That means that C. trachomatis still may be responsible for neonatal pneumonia in about one third of patients with manifest symptoms where the chlamydial etiology was presumed. 


\begin{tabular}{|c|c|c|c|}
\hline Age (weeks) & $\begin{array}{c}\text { Female } \\
\text { Positive/Studied } \\
\text { (prevalence } \%)\end{array}$ & $\begin{array}{c}\text { Male } \\
\text { Positive/Studied } \\
\text { (prevalence } \%)\end{array}$ & $\begin{array}{c}\text { Total } \\
\text { Positive/Studied } \\
\text { (prevalence \%) }\end{array}$ \\
\hline$\leq \mathbf{4}$ & $8 / 16(50.0 \%)$ & $10 / 22(45.5 \%)$ & $18 / 38(47.4 \%)$ \\
$\mathbf{5 - 8}$ & $10 / 27(37.0 \%)$ & $14 / 51(27.5 \%)$ & $24 / 78(30.8 \%)$ \\
$\mathbf{9 - 1 2}$ & $3 / 29(10.3 \%)$ & $15 / 52(28.8 \%)$ & $18 / 81(22.2 \%)$ \\
$\mathbf{1 3 - 1 6}$ & $8 / 17(47.1 \%)$ & $12 / 20(60.0 \%)$ & $20 / 37(54.1 \%)$ \\
$\mathbf{1 7 - 2 0}$ & $5 / 14(35.7 \%)$ & $7 / 13(53.8 \%)$ & $12 / 27(44.4 \%)$ \\
$\mathbf{2 1}-\mathbf{2 4}$ & $1 / 1(100.0 \%)$ & $0 / 8(0.0 \%)$ & $1 / 9(11.1 \%)$ \\
$\mathbf{2 5 - 2 8}$ & $0 / 2(0.0 \%)$ & $0 / 3(0.0 \%)$ & $0 / 5(0.0 \%)$ \\
$\mathbf{2 8}<$ & $0 / 2(0.0 \%)$ & $2 / 5(40.0 \%)$ & $2 / 7(28.6 \%)$ \\
\hline Total & $35 / 108(32.4 \%)$ & $60 / 174(34.5 \%)$ & $95 / 282(33.7 \%)$ \\
\hline
\end{tabular}

Table 1. Prevalence of $C$. trachomatis IgM serum antibodies in newborns with pneumonia

\section{Therapy}

Topical treatments, like silver nitrate eye drops (Credé-prophylaxis), antibiotics, povidoneiodine solution or others are ineffective in neonatal conjunctivitis and are unnecessary when a systemic treatment is administered. As chlamydial conjunctivitis is considered a local manifestation of a systemic colonisation, a systemic treatment is required. The drug of choice for the treatment of neonatal conjunctivitis and pneumonia is erythromycin 50 $\mathrm{mg} / \mathrm{kg} /$ day orally divided into 4 doses for 14 and for 21 days, respectively (Mårdh, 2002). The most serious complication of oral erythromycin treatment may be the development of hypertrophic pyloric stenosis. Another disadvantage of this regimen is a relatively high failure rate that was found $20 \%$ in cases of opthtalmia neonatorum or pneumonia. Therapeutic efficacy could be reliably controlled only by culture as nucleic acid detection techniques may give positive results detecting non-viable microbes up to 3 weeks after an adequate therapy, so the latter ones are inadequate for that purpose. A poor recovery rate was observed especially in too short (10 days or less) or too low-dose regimens. In recurring or persistent infections a second or even a third course of erythromycin may be needed (Miller, 2006; Quirke \& Cullinane, 2008; Zar, 2005).

Newer macrolide drugs, such as clarithromycin, roxithromycin or azithromycin may be effective therapeutic alternatives as well. The latter can have several advantages of use, such as a lower incidence of adverse effects, better tolerability and easier dosage regimen, however there is little data available about experiences in neonates. The specific diagnosis of C. trachomatis infection assists not only in the management of an infant's illness but also confirms the need for treating the mother and her sexual partner(s).

\section{Prevention}

Preventing neonatal morbidity caused by $C$. trachomatis can be achieved by different levels of probability of infection, but none of the interventions target the newborns directly. As detailed above, neither local nor systemic administration of drugs on neonates proved to be reliable and efficient means of prevention. Related to the vertical nature of transmission, chlamydiae should be eradicated from female organisms before and/or during pregnancy. Currently the CDC, the US Preventive Services Task Force (USPSTF), and numerous other 
professional medical associations recommend an annual chlamydia screening for all sexually active females aged $<25$ years and for females aged $\geq 25$ years if they are at increased risk for infection (CDC, 2011).

The maternal testing for $C$. trachomatis and the treatment of infections is invaluable in reducing perinatal transmission. The newest STD Treatment Guidelines (CDC, 2010) suggest that a test for $C$. trachomatis should be routinely performed at the first prenatal visit on all pregnant women. Women aged less than 25 years and those at increased risk (i.e., having a new sexual partner or multiple sexual partners) should be retested during the third trimester to prevent maternal postnatal complications and to reduce infant morbidity. Women with chlamydial infection diagnosed during the first trimester should be controlled for chlamydial eradication (preferably by NAAT) 3 weeks after the completion of the therapy AND they should be retested 3 months after the treatment, preferably before the third trimester.

In the absence of a comprehensive screening during pregnancy, clinicians should have a higher index of suspicion for neonatal and maternal chlamydial infections when the possibility of infection cannot be excluded. Infants born to mothers with untreated chlamydiae are at an even higher risk of infection but instead of a prophylactic therapy, a long-term observation is indicated as the efficacy of such prophylaxis is not confirmed yet. In the case of developing suspicious symptoms, children should be examined and given an adequate treatment (Darville, 2005).

Routine antenatal screening is the optimal solution not only for preventing neonatal chlamydial infections but also postpartum/chronic maternal complications. Maternal carriage always indicates the screening and treatment of sexual partner(s) as reinfection may occur at any time. Treatment should be given for the improvement of the pregnancy outcome as C. trachomatis may be related to perinatal complications, premature labor, and respiratory insufficiency of preterm infants too. As a first-line antichlamydial drug, azithromycin is recommended for treating urogenital infection in pregnancy, while amoxicillin may be a safe and well tolerable alternative regimen (Miller, 2006). The eradication of the pathogen may result in a decreasing rate of adverse pregnancy outcomes as reported by several studies (Allaire et al, 1995; Rastogi, 2003; Zar, 2005). The end of the second trimester is likely to be the most optimal time for screening pregnant women, if it is limited to only one occasion. In recognised infections an adequate therapy may still prevent the adverse pregnancy outcomes, while there is only 3 months left to potentially acquire the pathogen (Mårdh, 2002).

\section{Conclusion}

C. trachomatis represents an important STI pathogen among adults having also the ability to cause frequent neonatal infections. Neonatal inclusion conjunctivitis and infantile pneumonia may give rise to diagnostic and therapeutic difficulties. The aspecific, often mild-moderate symptoms may delay the definitive diagnosis while the complete eradication of the pathogen can be achieved only by a repeated therapy in several documented cases.

Our aim was to demonstrate the significant morbidity in infancy associated with $C$. trachomatis infection. Maternal C. trachomatis infection may cause various fetal or neonatal 
complications, and also indicates the urogenital presence of the pathogen of the mother and of her sexual partner(s). As $70-80 \%$ of the infected women are asymptomatic, the definitive diagnosis of neonatal infections may be occasionally the only epidemiologic clue that points to the infection of untreated mothers. The impact of this microorganism requires focused diagnostic approaches and an adequate antibiotic therapy not only applied to the affected infant but to both the mother and her partner(s) too.

Primary STI prevention should include the education and counselling of people about the risks of unsafe sexual behaviours, while secondary prevention consists of screening programs of asymptomatic persons at risk as well as the diagnosis and treatment of the infected patients. A national screening programme of pregnant women should also be established in Hungary, which concentrates on early diagnosis, effective treatment and close follow-up. The incidence of reinfection also needs to be investigated in further studies. Clinicians and neonatal nurses will need to be aware of the possibility of $C$. trachomatis infection, as early treatment rather than prevention seems to remain the best strategy for now, which will eventually lead to a decline in the incidence of perinatal chlamydial infection. The objective of this chapter has been to draw the attention to these infantile infections, which especially when overlooked, may still manifest as a severe condition that needs hospitalisation.

\section{Acknowledgements}

We express thanks to the laboratory staff: Ms. Éva Kelemen and Ms. Zsuzsa Kertész for their precise technical assistance, and to István Németh, $\mathrm{PhD}$ for ensuring an excellent, rapid analysis of our laboratory results.

\section{References}

Assadian, O. et al. (2002). Prophylaxis of ophthalmia neonatorum--a nationwide survey of the current practice in Austria. Wiener Klinische Wochenschrift, Vol. 114, No. 5-6, pp. 194-199

Attenburrow, A. A. \& Barker, C. M. (1986). Chlamydial pneumonia in the low birthweight neonate. Archives of Disease in Childhood, Vol. 60, No. 12, pp. 1169-1172

Baud, D.; Regan, L. \& Greub, G. (2008). Emerging role of Chlamydia and Chlamydia-like organisms in adverse pregnancy outcomes. Current Opinion in Infectious Diseases, Vol. 21, No. 1, pp. 70-76

Bell, T.A. et al (1992). Chronic Chlamydia trachomatis infections in infants. JAMA: The Journal of the American Medical Association, Vol. 267. No. 3, pp. 400-402

Bell, T.A. et al. (1994). Risk of perinatal transmission of Chlamydia trachomatis by mode of delivery. The Journal of Infection, Vol. 29, No. 2, pp. 165-169

Bertsche, A. et al. (2008). An unusual manifestation of a neonatal Chlamydia trachomatis infection. Journal of Child Neurology, Vol. 23, No. 8, pp. 948-949

Black-Payne, C., Bocchini J.A. Jr. \& Cedotal, C. (1989). Failure of erythromycin ointment for postnatal ocular prophylaxis of chlamydial conjunctivitis. The Pediatric Infectious Disease Journal, Vol. 8, No. 8, pp. 491-495 
Blas, M.M. et al. (2007). Pregnancy outcomes in women infected with Chlamydia trachomatis: a population-based cohort study in Washington State. Sexually Transmitted Infections, Vol. 83, No. 4, pp. 314-318

Boo, N.Y. (2008). Current understanding of congenital pneumonia. Pediatric Health, Vol. 2, No. 5, pp. 563-569

Brocklehurst, P. \& Rooney, G. (2007). Interventions for treating genital Chlamydia trachomatis infection in pregnancy. Cochrane Database of Systematic Reviews, Issue 4. Art. No.: CD000054. DOI: 10.1002/14651858.CD000054.

Chen, C. J. et al. (2007). Characteristics of Chlamydia trachomatis infection in hospitalized infants with lower respiratory tract infection. Journal of Microbiology, Immunology, and Infection, Vol.40, No.3, pp.255-259.

Centers for Disease Control and Prevention. (2010). Sexually Transmitted Diseases Treatment Guidelines. Morbidity and Mortality Weekly Report, Recommendations and Reports, No. 59, No. 12, pp. 1-114

Centers for Disease Control and Prevention. (2011). CDC Grand Rounds: Chlamydia Prevention: Challenges and Strategies for Reducing Disease Burden and Sequelae. Morbidity and Mortality Weekly Report, Recommendations and Reports, Vol. 60, No. 12, pp. 370-373

Colarizi, P. et al. (1996). Chlamydia trachomatis-associated respiratory disease in very early neonatal period. Acta Paediatrica, Vol. 85, No. 8, pp. 991-994

Dannevig, L.; Straume B. \& Melby, K. (1992). Ophthalmia neonatorum in northern Norway. II. Microbiology with emphasis on Chlamydia trachomatis. Acta Ophthalmologica, Vol. 70, No. 1, pp. $19-25$

Darling, E.K. \& McDonald, H. (2010). A meta-analysis of the efficacy of ocular prophylactic agets used for the prevention of gonococcal and chlamydial opthhalmia neoatorum. Journal of Midwifery \& Women's Health, Vol. 55, No. 4, pp. 319-327

Darville, T. (2005). Chlamydia trachomatis infections in neonates and young children. Seminars in Pediatric Infectious Diseases. Vol. 16, No. 4, pp. 235-244.

Da Silva, O.; Gregson, D. \& Hammerberg, O. (1997). Role of Ureaplasma urealyticum and Chlamydia trachomatis in development of bronchopulmonary dysplasia in very low birth weight infants. The Pediatric Infectious Disease Journal, Vol. 16, No. 4, pp. 364369

De Greeff, S.C. et al. (2010). Pertussis Disease Burden in the Household: How to Protect Young Infants. Clinical Infectious Diseases, Vol. 50, No. 10, pp. 1339-1345

Di Bartolomeo, S. et al. (2001). Incidence of Chlamydia trachomatis and other potential pathogens in neonatal conjunctivitis. International Journal of Infectious Diseases, Vol. 5, No. 3, pp. 139-143

Djukić, S. et al. (1996). Intra-amniotic Chlamydia trachomatis infection. Gynecologic and Obstetric Investigation, Vol. 42, No. 2, pp. 109-112

Dong, Z.W. et al. (1998). Detection of Chlamydia trachomatis intrauterine infection using polymerase chain reaction on chorionic villi. International Journal of Gynecology $\mathcal{E}$ Obstetrics, Vol. 61, No. 1, pp. 29-32.

Duke, T. (2005). Neonatal pneumonia in developing countries. Archives of Disease in Childhood. Fetal and Neonatal Edition, Vol. 90, No. 3, pp. 211-219 
Ejzenberg, B. et al. (1996). Aerobic bacteria, Chlamydia trachomatis, Pneumocystis carinii and Cytomegalovirus as agents of severe pneumonia in small infants. Revista do Instituto de Medicina Tropical de São Paul, Vol. 38, No. 1, pp. 9-14

European Centre for Disease Prevention and Control (2011). Sexually transmitted infections in Europe, 1990-2009. Stockholm: ECDC

Gallo Vaulet, L. et al. (2010). Distribution study of Chlamydia trachomatis genotypes in symptomatic patients in Buenos Aires, Argentina: association between genotype $\mathrm{E}$ and neonatal conjunctivitis. BMC Research Notes, Vol. 3, No. 34, pp. 1-6

Garland, S.M. \& Bowman, E.D. (1996). Role of Ureaplasma urealyticum and Chlamydia trachomatis in lung disease in low birth weight infants, Pathology, Vol. 28, No. 3, pp. 266-269

Gencay, M. et al. (2001). Chlamydia trachomatis infection in mothers with preterm delivery and in their newborn infants. APMIS: Acta Pathologica, Microbiologica, et Immunologica Scandinavica, Vol. 109, No. 9, pp. 636-640

Givner, L.B. et al. (1981). Chlamydia trachomatis infection in infant delivered by caesarean section. Pediatrics, Vol. 68, No. 3, pp. 420-421.

Herieka, E. \& Dhar, J. (2001). Acute neonatal respiratory failure and Chlamydia trachomatis. Sexually Transmitted Infections, Vol. 77, No. 2,pp. 135-136

Isobe, K. et al. (1996). Serotyping Chlamydia trachomatis from inclusion conjunctivitis by polymerase chain reaction and restriction length polymorphism analysis. Japanese Journal of Ophthamology, Vol. 40, No. 2, pp. 279-285

Iskandar, N.M. \& Naguib, M.B. (1998). Chlamydia trachomatis: An underestimated cause for rhinitis in neonates. International Journal of Pediatric Otorhinolaryngology, Vol. 42, No. 3, pp. 233-237

Jadavji, T. et al. (1997). A practical guide for the diagnosis and treatment of pediatric pneumonia. Canadian Medical Association Journal, Vol. 156, No. 5, pp. 703-711.

Jain, S. (1999). Perinatally aquired Chlamydia trachomatis associated morbidity in young infants. Journal of Maternal-Fetal Medicine, Vol. 8, No. 3, pp. 130-133

Jarvis, V. N.; Levine, R. \& Asbell P.A. (1987). Ophthalmia neonatorum: study of a decade of experience at the Mount Sinai Hospital. British Journal of Ophtalmology, Vol. 71, No. 4, pp. $295-300$

Johnson, R. E. et al. (2002). Screening Tests To Detect Chlamydia trachomatis and Neisseria gonorrhoeae Infections. Morbidity and Mortality Weekly Report, Recommendations and Reports, Vol. 51, No. 15, pp. 1-37

Jones, B.R.; Collier, L.H. \& Smith, C.H. (1959). Isolation of virus from inclusion blennorrhoea. Lancet Vol. 1, No. 7079, pp. 902-905.

Klein, J.O. \& Barnett, E.D. (1998). Neonatal pneumonia. Seminars in Pediatric Infectious Diseases, Vol. 9, No. 3, pp. 212-216

Krohn, M. A. et al. (1993). The bacterial etiology of conjunctivitis in early infancy. American Journal of Epidemiology, Vol. 138, No. 5, pp. 326-332

Lamont, R.F. et al. (1987). The role of mycoplasmas, ureaplasmas and chlamydiae in the genital tract of women presenting in spontaneous early preterm labour. Journal of Medical Microbiology, Vol. 24, No. 3, pp. 253-257

Locksmith, G. \& Duff, P. (2001). Infection, antibiotics, and preterm delivery. Seminars in Perinatology, Vol. 25, No. 5, pp. 295-309 
López-Hurtado, M. et al. (1999). Prevalence of Chlamydia trachomatis in newborn infants with respiratory problems. Revista latinoamericana de microbiología, Vol. 41, No. 4, pp. 267272

Magon, T. et al. (2005). The PCR assessed prevalence of Chlamydia trachomatis in aborted tissues. Medycyna wieku rozwojowego, Vol. 9, No. 1, pp. 43-48.

Mahony, J.B. et al. (1986). Accuracy of immunoglobulin M immunoassay for diagnosis of chlamydial infections in infants and adults. Journal of Clinical Microbiology, Vol. 24, No. 5, pp. 731-735

Mårdh, P.A. (2002). Influence of infection with Chlamydia trachomatis on pregnancy outcome, infant health and life-long sequelae in infected offspring. Best Practice $\mathcal{E}$ Research Clinical Obstetrics \& Gynaecology, Vol. 16, No. 6, pp. 847-864.

Marín Gabriel, M.A. et al (2004). Respiratory infection due to Chlamydia trachomatis in infants. Clinical presentation and outcome in 18 patients. Anales de pediatría (Barcelona, Spain : 2003), Vol. 60, No. 4, pp. 349-353

Maxwell, G.L. (1993). Preterm premature rupture of membranes. Obstetrical \& Gynecological Survey, Vol. 48, No. 8, pp. 576-583.

Miller, K. E. (2006). Diagnosis and treatment of Chlamydia trachomatis infection. American Family Physician, Vol. 73, No. 8, pp. 1411-1416

Mohile, M. et al. (2002). Microbiological study of neonatal conjunctivitis with special reference to Chlamydia trachomatis. Indian Journal of Ophthalmology, Vol. 50, No. 4, pp. 295-299

Nsanze, H. et al. (1996). Ophthalmia neonatorum in the United Arab Emirates. Annals of Tropical Paediatrics, Vol. 16, No. 1, pp. 27-32.

Numazaki, K. et al. (1984). Pneumonia due to Chlamydia trachomatis in Japanese Infants. The Tohoku Journal of Experimental Medicine, Vol. 143, No. 4, pp. 413-420

Numazaki, K. et al. (1986). Chronic respiratory disease in premature infants caused by Chlamydia trachomatis. Journal of Clinical Pathology, Vol.39, No.1, pp.84-88

Numazaki, K.; Wainberg, M.A. \& McDonald, J. (1989). Chlamydia trachomatis infections in infants. Canadian Medical Association Journal, Vol. 140, No.6, pp.615-622.

Numazaki, K.; Asanuma, H. \& Niida, Y. (2003). Chlamydia trachomatis infection in early neonatal period. BMC Infectious Diseases, Vol. 3, No. 2, pp. 1-5

Numazaki, K. (2004). Current problems of perinatal Chlamydia trachomatis infections. Journal of Immune Based Therapies and Vaccines, Vol. 2, No. 1, pp. 1-7

Odeh, M. \& Oliven, A. (1992). Chlamydial Infections of the Heart. European Journal of Clinical Microbiology \& Infectious Diseases, Vol. 11, No. 10, pp. 885-893

Ostaszewska-Puchalska, I. et al. (2005). Chlamydia trachomatis infections in women with adverse pregnancy outcome. Medycyna wieku rozwojowego, Vol. 9, No. 1, pp. 49-56

Peipert, J.F. (2003). Clinical practice. Genital chlamydial infections. The New England Journal of Medicine, Vol. 349, No. 25, pp. 2424-2430.

Pellowe, C. \& Pratt, R.J. (2006). Neonatal conjunctivitis and pneumonia due to chlamydia infection. Infant, Vol. 2, No. 1, pp. 16-17

Persson, K. et al. (1983). Neonatal chlamydial eye infection: an epidemiological and clinical study. British Journal of Ophtalmology, Vol. 67, No. 10, pp. 700-704

Petrovay, F. et al. (2009). Genotyping of Chlamydia trachomatis from the endocervical specimens of high-risk women in Hungary. Journal of Medical Microbiology, Vol. 58, No. 6, pp. $760-764$ 
Pierce, J.M.; Ward, M.E. \& Seal, D.V. (1982). Ophthalmia neonatorum in the 1980's: incidence, aetiology and treatment. British Journal of Ophthalmology, Vol. 66, No. 11, pp. 728-731

Preece, P.M.; Anderson, J.M. \& Thompson, R.G. (1989). Chlamydia trachomatis infection in infants: a prospective study. Archives of Disease in Childhood, Vol. 64, No. 4, pp. 525529.

Prentice, J.M.; Hutchinson, G. R. \& Taylor-Robinson, D. (1977). A microbiological study of neonatal conjunctivae and conjunctivitis. British Journal of Ophthalmology, Vol. 61, No. 9, pp. 601-607

Quirke, M. \& Cullinane, A. (2008). Recent trends in chlamydial and gonococcal conjunctivitis among neonates and adults in an Irish hospital. International Journal of Infectious Diseases, Vol. 12, No. 4, pp. 371-373

Rastogi, S. et al. (1999). Chlamydia trachomatis infection in pregnancy: risk factor for an adverse outcome. British Journal of Biomedical Science. Vol. 56, No. 2, pp. $94-98$

Rastogi, S. et al. (2003). Effect of treatment for Chlamydia trachomatis during pregnancy. International Journal of Gynecology \& Obstetrics, Vol. 80, No. 2, pp. 129-37

Ratelle, S. et al. (1997). Neonatal chlamydial infections in Massachusetts, 1992-1993. American Journal of Preventive Medicine, Vol. 13, No. 3, pp. 221-224

Rees, E. et al. (1981). Persistence of chlamydial infection after treatment of neonatal conjunctivitis. Archives of Diseases in Childhood, Vol. 56, No. 3, pp. 193-198

Rezeberga, D. et al. (2008). Placental histological inflammation and reproductive tract infections in a low risk pregnant population in Latvia. Acta Obstetricia et Gynecologica, Vol. 87, No. 3, pp. 360-365

Ringel, R.E. et al. (1983). Myocarditis as a complication of infantile Chlamydia trachomatis pneumonitis. Clinical Pediatrics, Vol. 22, No. 9, pp. 631-633

Rosenman, M. B. et al (2003). Oral erythromycin prophylaxis vs watchful waiting in caring for newborns exposed to Chlamydia trachomatis. Archives of Pediatrics and Adolescent Medicine, Vol. 157, No. 6, pp. 565-571

Rours, G.I. et al. (2008). Chlamydia trachomatis as a cause of neonatal conjunctivitis in Dutch infants. Pediatrics, Vol. 121, No. 2, pp. 321-326

Rours, G.I. et al. (2009). Chlamydia trachomatis respiratory infection in Dutch infants. Archives of Diseases in Childhood, Vol. 94, No. 9, pp. 705-707

Rours, G.I. et al. (2011). Chlamydia trachomatis infection during pregnancy associated with preterm delivery: a population-based prospective cohort study. European Journal of Epidemiology, Vol. 26, No. 6, pp. 493-502

Salpietro, C.D. et al. (1999). Chlamydia trachomatis conjunctivitis in the newborn. Archives de pédiatrie, Vol. 6, No. 3, pp. 317-320

Sandström, I. (1987). Etiology and diagnosis of neonatal conjunctivitis. Acta Paediatrica Scandinavica, Vol. 76, No. 2, pp. 221-227

Schachter, J. et al. (1975). Pneumonitis following inclusion blennorrhoea. The Journal of Pediatrics, Vol. 87, No. 5, pp. 779-780

Schachter, J. et al. (1979). Infection with Chlamydia trachomatis: involvement of multiple anatomic sites in neonates. The Journal of Infectious Diseases, Vol. 139, No. 2, pp. 232234. 
Schachter, J. et al. (1986). Prospective study of perinatal transmission of Chlamydia trachomatis. JAMA: The Journal of the American Medical Association, Vol. 255. No. 24, pp. 3374-3377

Schachter, J. \& Stamm, W.E. (1995). Chlamydia, In: Manual of clinical microbiology, Murray, P.R. et al, pp. 669-677, American Society for Microbiology, Washington, D.C.

Schaefer, C. et al. (1985). Illnesses in infants born to women with Chlamydia trachomatis infection. A prospective study. American journal of diseases of children, Vol. 139, No. 2, pp. 127-133

Schaller, U. \& Klauss, V. (2001). Is Crede's prophylaxis for ophthalmia neonatorum still valid? Bulletin of the World Health Organisation, Vol. 79, No. 3, pp. 262-263

Silva, L. R. et al. (2008). Current usefulness of Credé's method of preventing neonatal ophthalmia. Annals of Tropical Paediatrics, Vol. 28, No. 1, pp. 45-48

Solberg, R.; Meberg, A. \& Schøyen, R. (1991). Neonatal conjunctivitis in a nursery and a neonatal unit. Tidsskrift for den Norske lægeforening, Vol. 111, No. 10, pp. 1230-1232

Sollecito, D. et al. (1992). Chlamydia trachomatis in neonatal respiratory distress of very preterm babies; biphasic clinical picture. Acta Paediatrica, Vol. 81, No. 10, pp. 788791

Stagno, B. et al. (1981). Infant pneumonitis associated with cytomegalovirus, Chlamydia, Pneumocystis and Ureaplasma: a prospective study. Pediatrics, Vol. 68, No. 3, pp. 322-329

Stenberg, K. \& Mårdh, P.A. (1990). Chlamydial conjunctivitis in neonates and adults. History, clinical findings and follow-up. Acta Ophthalmologica, Vol. 68, No. 6, pp. 651-657

Thompson, C; Macdonald, M. \& Sutherland, A. (2001). A family cluster of Chlamydia trachomatis infection, British Medical Journal, Vol. 322, No. 7300, pp. 1472-1474

Valencia, C. et al. (2000). Prevalence of the Chlamydia trachomatis in neonatal conjunctivitis determination by indirect fluorescence and gene amplification. Revista médica de Chile, Vol. 128, No. 7, pp. 758-765

Vieira, R.A. et al. (2003). Clinical and laboratoty study of newborns with lower respiratory tract infection due to respiratory viruses. The Journal of Maternal-Fetal and Neonatal Medicine, Vol. 13, No. 5, pp. 341-350

Were, F.N. et al. (2002). Chlamydia as a cause of late neonatal pneumonia at Kenyatta National Hospital, Nairobi. East African Medical Journal, Vol. 79, No. 9, pp. 476-479

Webley, W.C. et al. (2009). Occurrence of Chlamydia trachomatis and Chlamydia pneumoniae in paediatric respiratory infections. The European Respiratory Journal, Vol. 33, No. 2, pp. 360-367

Wilson, J. S. et al. (2002). A systematic review of the prevalence of Chlamydia trachomatis among European women. European Society of Human Reproduction and Embryology,Vol. 8, No. 4, pp. 385-894.

Winceslaus, J. et al. (1987). Diagnosis of ophthalmia neonatorum. British Medical Journal, Vol. 295, No. 6610, pp. 1377-1379

Yoshida, H. et al. (2000). Reversal of intra-amniotic Chlamydia trachomatis antigen status. Gynecologic and Obstetric Investigation, Vol. 50, No. 4, pp. 278-280.

$\mathrm{Yu}$, J. et al. (2009). Vertical transmission of Chlamydia trachomatis in Chongqing China. Current Microbiology, Vol. 58, No. 4, pp. 315-320 
Zar, H.J. et al. (1999). Chlamydia trachomatis lower respiratory tract infection in infants. Annals of Tropical Paediatrics, Vol. 19, No.1, pp. 9-13

Zar, H.J. (2005). Neonatal chlamydial infections: prevention and treatment. Pediatric Drugs, Vol. 7, No. 2, pp. 103-110

Zuppa, A.A. et al. (2011). Ophthalmia neonatorum: what kind of prophylaxis? The Journal of Maternal-Fetal and Neonatal Medicine, Vol. 24, No. 6, pp. 769-773 


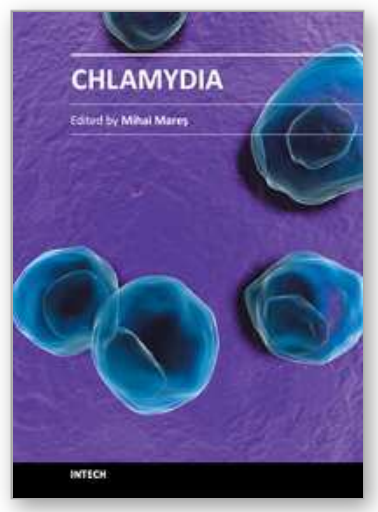

\author{
Chlamydia \\ Edited by Prof. Mihai Mares
}

ISBN 978-953-51-0470-4

Hard cover, 358 pages

Publisher Intech

Published online 30, March, 2012

Published in print edition March, 2012

Nowadays, Chlamydia still represents a redoubtable pathogen. Among its consequences, the blindness in children and severe impairment of reproductive health in adults are the most mutilating. Worldwide, it is estimated that six million of people suffer from post-trachoma blindness and almost 90 million become sexually infected each year. Due to its silent evolution and sexually transmission, the chlamydial infection can occur in anyone. The book "Chlamydia - A Multifaceted Pathogen" contains an updated review of all-important issues concerning the chlamydial infection. It comprises 18 chapters grouped in four major parts dealing with etiology and pathogenicity, clinical aspects, diagnosis and prevention. The new molecular data about the pathogenicity and the exhaustive presentation of clinical findings bring novelty to the book and improve our knowledge about Chlamydia induced diseases.

\title{
How to reference
}

In order to correctly reference this scholarly work, feel free to copy and paste the following:

Eszter Balla and Fruzsina Petrovay (2012). Chlamydia trachomatis Infections in Neonates, Chlamydia, Prof. Mihai Mares (Ed.), ISBN: 978-953-51-0470-4, InTech, Available from:

http://www.intechopen.com/books/chlamydia/chlamydia-trachomatis-infections-in-neonates

\section{INTECH}

open science | open minds

\section{InTech Europe}

University Campus STeP Ri

Slavka Krautzeka 83/A

51000 Rijeka, Croatia

Phone: +385 (51) 770447

Fax: +385 (51) 686166

www.intechopen.com

\section{InTech China}

Unit 405, Office Block, Hotel Equatorial Shanghai

No.65, Yan An Road (West), Shanghai, 200040, China 中国上海市延安西路65号上海国际贵都大饭店办公楼 405 单元

Phone: +86-21-62489820

Fax: +86-21-62489821 
(C) 2012 The Author(s). Licensee IntechOpen. This is an open access article distributed under the terms of the Creative Commons Attribution 3.0 License, which permits unrestricted use, distribution, and reproduction in any medium, provided the original work is properly cited. 